\title{
WEAK COMPACTNESS IN LOCALLY CONVEX SPACES
}

\author{
J. D. PRYCE
}

1. Introduction. A recently published paper of R. C. James [1] proves the following Theorem: A weakly closed set $C$ in a Banach space $B$ is weakly compact if and only if every bounded linear functional on $B$ attains its supremum on $C$ at some point of $C$. The proof given by James is rather long and involved: the following, while not employing any basically different ideas, is a simpler version and extends the theorem with no extra effort to deal with a locally convex linear topological space rather than a Banach space, using the Eberlein criterion for weak compactness (see e.g. [2, p. 159]).

\section{The result.}

THEOREM. Let $C$ be a weakly closed bounded subset of the real and complete locally convex linear topological space $E$. Then $C$ is weakly compact if and only if given any element $f$ of the dual $E^{*}$ of $E$, there is $x \in C$ such that $f(x)=\sup \{f(u): u \in C\}$.

Corollary. The hypothesis that $E$ be complete may be replaced by the hypothesis that the closed convex hull of $C$ be complete (in the original topology of E).

Proof. The implication one way is elementary: namely, suppose $C$ is weakly compact and $f$ any element of $E^{*}$. Then by the definition of the weak topology $f$ is continuous on $C$ in the weak topology and so attains its bounds.

We prove the implication the other way by assuming that $C$ is not weakly compact, and constructing a continuous linear functional which does not attain its supremum on $C$ at any point of $C$. The proof of this fact is divided up into a series of lemmas.

Lemma 1 . There is a sequence $\left(z_{n}\right)$ of points in $C$ and a sequence $\left(f_{n}\right)$ of elements of $E^{*}$ such that $\left\{f_{n}\right\}$ is an equicontinuous set and the limits $\lim _{i} \lim _{j} f_{i}\left(z_{j}\right)$ and $\lim _{j} \lim _{i} f_{i}\left(z_{j}\right)$ exist and are unequal.

For the proof of this result, which is Eberlein's celebrated compactness theorem, see [2], where the result is stated on p. 159.

We now introduce some notation. Since we shall not be dealing only with functionals on $E$ that are linear, we denote by $F$ the set of all real-valued continuous functions on $E$ which are positive-homogeneous,

Received by the editors March 1, 1965. 


$$
f(\alpha x)=\alpha f(x)
$$

Since for each $f \in F$, there is a neighbourhood $U$ of 0 in $E$ such that $|f(x)|=|f(x)-f(0)| \leqq 1 \quad(x \in U)$, elements of $F$ are bounded on bounded sets. Note that $E^{*}$ is a subspace of $F$. We give $F$ the weak* topology $w(F, E)$ a pointwise convergence on $E$; this makes $E^{*}$ a weak*-closed subspace.

We define

$$
p(f)=\sup \{f(x): x \in C\}
$$

The functional $p$ is finite-valued and has the following properties:

(i) $p$ is sublinear, i.e. $p(\lambda f)=\lambda p(f)$ for $\lambda \geqq 0$ and

$$
p(f+g) \leqq p(f)+p(g) \text {. }
$$

(ii) Since

$$
p(f) \leqq p(g)+p(f-g)
$$

and

$$
p(g) \leqq p(f)+p(g-f)
$$

we have

$$
-p(g-f) \leqq p(f)-p(g) \leqq p(f-g) .
$$

(iii) If $A \subseteq F$ and $A$ is equicontinuous then $p$ is bounded on $A$, for there is a neighbourhood $U$ of 0 in $E$ such that $|f(x)| \leqq 1$ for all $x \in U, f \in A$, and $C$ is absorbed by $U$.

We also define $P(f)=\sup \{|f(x)|: x \in C\}$ for $f \in F$. The functional $P$ is a seminorm inducing on $F$ the topology of uniform convergence on $C$.

Let $\left(f_{i}\right)$ be a sequence in $F$ which is equicontinuous at each point of $E$, and define functions $G_{-}=\lim \inf f_{i}, G^{-}=\lim \sup f_{i}$ by

$$
G_{-}(x)=\liminf f_{i}(x), \quad G^{-}(x)=\lim \sup f_{i}(x) \quad(x \in E) .
$$

Given $x_{0}$ and $\epsilon$ there is a neighbourhood $U$ of $x_{0}$ such that

$$
\sup \left\{\left|f_{i}(x)-f_{i}\left(x_{0}\right)\right|: i=1,2, \cdots ; x \in U\right\} \leqq \epsilon .
$$

Applying this and the relation $\left|\lim \inf f_{i}(x)-\lim \inf f_{i}\left(x_{0}\right)\right|$ $\leqq \sup _{i}\left|f_{i}(x)-f_{i}\left(x_{0}\right)\right|$ first to 0 and then to an arbitrary $x_{0}$ we see that $G_{-}$, and similarly $G^{-}$, is everywhere finite and continuous. It is clearly positive-homogeneous and so belongs to $F$.

Lemma 2. Let $\left(f_{i}\right)$ be a sequence in $F$ equicontinuous at each point. Let the topology on $F$ be that of the seminorm $P$, and let $X$ be any subset of $F$ which is separable in the relative topology. Then there is a subse- 
quence $\left(G_{i}\right)$ of the $f_{i}$ such that if $G_{-}=\lim \inf G_{i}, G^{-}=\lim \sup G_{i}$, we have $p\left(f-G_{-}\right)=p\left(f-G^{-}\right)$for all $f \in X$.

Proof. Let $\left(\omega_{k}\right)$ be a dense sequence in $X$. By replacing it by the sequence $\omega_{1}, \omega_{1}, \omega_{2}, \omega_{1}, \omega_{2}, \omega_{3}, \omega_{1}, \omega_{2}, \omega_{3}, \omega_{4}, \cdots$, we can assume that each point of $X$ is a cluster point of the sequence. We now apply a diagonal process, inductively defining points $x_{n}$ and sequences $\left(f_{i}^{n}: i=1,2, \cdots\right)$ as follows:

For $n=1$ choose $x_{1} \in C$ so that

$$
\omega_{1}\left(x_{1}\right)-\lim \inf f_{i}\left(x_{1}\right)>p\left(\omega_{1}-\liminf f_{i}\right)-\frac{1}{2},
$$

while for $n>1$ choose $x_{n} \in C$ so that

$$
\omega_{n}\left(x_{n}\right)-\liminf _{i} f_{i}^{n-1}\left(x_{n}\right)>p\left(\omega_{n}-\liminf _{i} f_{i}^{n-1}\right)-2^{-n},
$$

and $\left(f_{i}^{n}: i=1,2, \cdots\right)$ as a subsequence of $\left(f_{i}^{n-1}: i=2,3, \cdots\right)$, so that

$$
f_{i}^{n}\left(x_{n}\right) \text { converges to } \liminf _{i} f_{i}^{n-1}\left(x_{n}\right) \quad \text { as } i \rightarrow \infty .
$$

(Note that $f_{1}^{n-1}$ thus does not occur as a member of $\left(f_{i}^{n}\right)$.) Now define $G_{k}=f_{1}^{z}$. Since $\left(G_{n}, G_{n+1}, \cdots\right)$ is for each $n$ a subsequence of $\left(f_{1}^{n}, f_{2}^{n}, \cdots\right)$ we have, if $G_{-}, G^{-}$denote $\lim \inf G_{k}, \lim \sup G_{k}$, for every $n$,

$$
\lim _{k} G_{k}\left(x_{n}\right) \text { exists and equals } \lim _{i} f_{i}^{n}\left(x_{n}\right) ;
$$

(ii) $\omega_{n}\left(x_{n}\right)-G_{-}\left(x_{n}\right)=\omega_{n}\left(x_{n}\right)-\lim _{i} f_{i}^{n}\left(x_{n}\right)=\omega_{n}\left(x_{n}\right)-\lim _{i} \inf _{i}^{n-1}\left(x_{n}\right)$

$$
\begin{aligned}
& >p\left(\omega_{n}-\liminf _{i} f_{i}^{n-1}\right)-2^{-n} \\
& \geqq p\left(\omega_{n}-\underset{k}{\lim \inf } G_{k}\right)-2^{-n}=p\left(\omega_{n}-G_{-}\right)-2^{-n} .
\end{aligned}
$$

Now let $f$ be an element of $X$. Because of the cluster point property of the $\omega_{k}$, given any $\epsilon>0$ there is $n$ such that (i) $2^{-n}<\epsilon$ and (ii) for all $x$ in $C,\left|f(x)-\omega_{n}(x)\right|<\epsilon$. Then we have

$$
\begin{aligned}
p\left(f-G_{-}\right) & \leqq \epsilon+p\left(\omega_{n}-G_{-}\right) \\
& <2 \epsilon+\omega_{n}\left(x_{n}\right)-G_{-}\left(x_{n}\right) \\
& =2 \epsilon+\omega_{n}\left(x_{n}\right)-G^{-}\left(x_{n}\right) \\
& <3 \epsilon+f\left(x_{n}\right)-G^{-}\left(x_{n}\right) \\
& \leqq 3 \epsilon+p\left(f-G^{-}\right) .
\end{aligned}
$$


Since $\epsilon$ is arbitrary we have $p\left(f-G_{-}\right) \leqq p\left(f-G^{-}\right)$; the opposite inequality is trivial and hence $p\left(f-G_{-}\right)=p\left(f-G^{-}\right)$. This proves the lemma.

Let $\left(f_{i}\right)$ now be the sequence of Lemma 1 and $X$ be the linear span of the $f_{i}$. In the $P$ topology $X$ is separable (e.g. take linear combinations of the $f_{i}$ with rational coefficients), so the conditions of Lemma 2 are satisfied, and we can by taking a subsequence assume that

$$
p\left(f-G_{-}\right)=p\left(f-G^{-}\right)
$$

where $G_{-}=\lim \inf f_{i}, G^{-}=\lim \sup f_{i}$. The double limit relation of Lemma 1 is not disturbed by this process. Further we can without loss of generality assume that $f_{k}\left(z_{j}\right)-\lim _{i} f_{i}\left(z_{j}\right)$ is for each $k$ eventually $\geqq r>0$, as $j$ tends to infinity, (by another application of the diagonal process).

Let $K_{n}$ denote the convex hull of $\left\{f_{n}, f_{n+1}, \cdots\right\}$ for $n=1,2, \cdots$. To keep the record straight, we have $F \supseteq E^{*} \supseteq X \supseteq K_{1} \supseteq K_{2} \supseteq \cdots$.

Lemma 3. For all $f \in K_{1}, p\left(f-G_{-}\right) \geqq r$.

Proof. Let $f$ be any element of $K_{1}$; then $f=\sum_{i=1}^{i} \lambda_{i} f_{n_{i}}$, where $\lambda_{i} \geqq 0$, and $\sum_{1}^{s} \lambda_{i}=1$. Then

$$
\begin{aligned}
p\left(f-G_{-}\right) & \geqq f\left(z_{j}\right)-G_{-}\left(z_{j}\right)=\sum_{1}^{s} \lambda_{i}\left\{f_{n_{i}}\left(z_{j}\right)-G_{-}\left(z_{j}\right)\right\} \\
& =\sum_{1}^{s} \lambda_{i}\left\{f_{n_{i}}\left(z_{j}\right)-\underset{r}{\lim } f_{r}\left(z_{j}\right)\right\} \\
& \geqq \sum_{1}^{s} \lambda_{i} r=r
\end{aligned}
$$

if we choose $j$ large enough.

Lemma 4. Let $Y$ be a linear space, and $\rho, \beta, \beta^{\prime}$ be strictly positive numbers. Let $A$ be a convex subset of $Y, u$ a point of $Y$, and $p$ a sublinear functional on $Y$. Suppose that

$$
\inf _{a \in A} p(u+\beta a)>\beta \rho+p(u) .
$$

Then there is a point $a_{0}$ in $A$ such that

$$
\inf _{b \in A} p\left(u+\beta a_{0}+\beta^{\prime} b\right)>\beta^{\prime} \rho+p\left(u+\beta a_{0}\right) .
$$

Proof. Choose any $x, y$ in $A$ and set $c=\left(\beta x+\beta^{\prime} y\right) /\left(\beta+\beta^{\prime}\right)$. Then $c \in A$ and $u+\beta x+\beta^{\prime} y=u+\left(\beta+\beta^{\prime}\right) c=\left(1+\beta^{\prime} / \beta\right)(u+\beta c)-\left(\beta^{\prime} / \beta\right) u$. From the hypothesis of the lemma,

$$
-p(u)=\beta \rho-\inf _{a \in A} p(u+\beta a)+\delta
$$


and by sublinearity,

$$
p\left(u+\beta x+\beta^{\prime} y\right) \geqq p\left(\left(1+\beta^{\prime} / \beta\right)(u+\beta c)\right)-p\left(\left(\beta^{\prime} / \beta\right) u\right) .
$$

Hence for fixed $a_{0}$ in $A$,

$$
\begin{aligned}
\inf _{b \in A} p\left(u+\beta a_{0}+\beta^{\prime} b\right) \\
\quad \geqq\left(1+\frac{\beta^{\prime}}{\beta}\right) \inf \left\{p(u+\beta c): c=\frac{\beta a_{0}+\beta^{\prime} b}{\beta+\beta^{\prime}}, b \in A\right\}-\frac{\beta^{\prime}}{\beta} p(u) \\
\geqq\left(1+\frac{\beta^{\prime}}{\beta}\right) \inf _{a \in A} p(u+\beta a)-\frac{\beta^{\prime}}{\beta} p(u) \\
\quad=\left(1+\frac{\beta^{\prime}}{\beta}\right) \inf _{a \in A} p(u+\beta a)+\frac{\beta^{\prime}}{\beta}\left(\beta \rho-\inf _{a \in A} p(u+\beta a)\right)+\frac{\beta^{\prime}}{\beta} \delta \\
=\beta^{\prime} \rho+\inf _{a \in A} p(u+\beta a)+\frac{\beta^{\prime}}{\beta} \delta .
\end{aligned}
$$

Thus if we choose $a_{0}$ so that $p\left(u+\beta a_{0}\right)<\inf _{a \in A} p(u+\beta a)+\left(\beta^{\prime} / \beta\right) \delta$ we obtain the required result.

Lemma 5. Let $\left(\beta_{n}\right)$ be an arbitrary sequence of strictly positive real numbers. Then there is a sequence $\left(g_{n}\right)$ in $F$ such that for all $n, g_{n} \in K_{n}$ and

$$
p\left[\sum_{1}^{n} \beta_{i}\left(g_{i}-G_{-}\right)\right]>\frac{1}{2} \beta_{n} r+p\left[\sum_{1}^{n-1} \beta_{i}\left(g_{i}-G_{-}\right)\right] .
$$

Proof. We use induction and Lemma 4.

For the first step, let $u=0, \beta=\beta_{1}, \beta^{\prime}=\beta_{2}$ and $A$ be the set $K_{1}-G_{-}$ $=\left\{f-G_{-}: f \in K_{1}\right\} ;$ and $p$ as already defined. Then $\inf _{f \in A} p(u+\beta f)$ $=\inf _{f \in K_{1}} p\left[\beta_{1}\left(f-G_{-}\right)\right] \geqq \beta_{1} r>\frac{1}{2} \beta_{1} r+p(u)$ by Lemma 3 , so the conditions of Lemma 4 are satisfied. Hence there is $g_{1} \in K_{1}$ such that

$$
\inf _{g \in K_{1}} p\left[\beta_{1}\left(g_{1}-G_{-}\right)+\beta_{2}\left(g-G_{-}\right)\right]>\frac{1}{2} \beta_{2} r+p\left[\beta_{1}\left(g_{1}-G_{-}\right)\right] \text {. }
$$

For the $n$th step, let $u=\sum_{1}^{n-1} \beta_{i}\left(g_{i}-G_{-}\right), \beta=\beta_{n}, \beta^{\prime}=\beta_{n+1}$ and $A$ be the set $K_{n}-G_{-}$. By the inductive hypothesis, and since $K_{n-1} \supseteq K_{n}$,

$$
\inf _{f \in A} p(u+\beta f) \geqq \inf \left\{p(u+\beta f): f \in K_{n-1}-G_{-}\right\}>\frac{1}{2} \beta_{n} r+p(u)
$$

and Lemma 4 gives $g_{n} \in K_{n}$ such that if $v=\sum_{1}^{n} \beta_{i}\left(g_{i}-G_{-}\right)$,

$$
\inf _{f \in A} p\left(v+\beta^{\prime} f\right)>\frac{1}{2} \beta^{\prime} r+p(v)
$$


which is the inductive hypothesis for $n$. The sequence $\left(g_{n}\right)$ then has the required property.

LeMma 6. There is $G_{0}$ in $E^{*}$ such that

(i) $\lim \inf g_{n}(x) \leqq G_{0}(x) \quad(x \in E)$,

(ii) $p\left(h-G_{0}\right)=p\left(h-G_{-}\right) \quad(h \in X)$.

Proof. The set $K_{1}$ is the convex hull of the equicontinuous sequence $\left(f_{n}\right)$, and thus the weak ${ }^{*}$-closure of $K_{1}$ in $E^{*}$ is weak ${ }^{*}$-compact. The sequence $\left(g_{n}\right)$ lies in $K_{1}$ and therefore has a weak* clusterpoint $G_{0}$ in $E^{*}$. Then for each $x$ in $E, G_{0}(x)$ is a cluster-point of the real number sequence $\left(g_{n}(x)\right)$, and so

$$
\lim \inf g_{n}(x) \leqq G_{0}(x) \leqq \lim \sup g_{n}(x),
$$

which establishes (i).

Next, since $g_{n} \in K_{n}, g_{n}(x)$ is a convex combination $\sum \lambda_{i} f_{m_{i}}(x)$, with the $m_{i}$ not less than $n$. It follows that there is at least one of the $m_{i}$ for which $f_{m_{i}}(x) \leqq g_{n}(x)$. In other words, given any $n$ there is $m \geqq n$ such that

$$
f_{m}(x) \leqq g_{n}(x),
$$

and so $G_{-}(x)=\lim \inf f_{n}(x) \leqq \lim \sup g_{n}(x)$.

A similar argument on the other side establishes $G^{-}(x) \geqq \lim \sup g_{n}(x)$. Combining our inequalities we have $G_{-}(x) \leqq G_{0}(x) \leqq G^{-}(x),(x \in E)$ and so

$$
p\left(h-G_{-}\right) \geqq p\left(h-G_{0}\right) \geqq p\left(h-G^{-}\right) \quad(h \in X) .
$$

The outer terms are equal and the lemma is proved.

COROLlary. The conclusion of Lemma 5 holds with $G_{-}$replaced by $G_{0}$.

Proof. Fix $n$ and let $\alpha=\beta_{1}+\cdots+\beta_{n}$. Then

$$
\begin{aligned}
p\left[\sum_{1}^{n} \beta_{i}\left(g_{i}-G_{0}\right)\right] & =\alpha p\left[\frac{1}{\alpha} \sum_{1}^{n} \beta_{i} g_{i}-G_{0}\right]=\alpha p\left[\frac{1}{\alpha} \sum_{1}^{n} \beta_{i} g_{i}-G_{-}\right] \\
& =p\left[\sum_{1}^{n} \beta_{i}\left(g_{i}-G_{-}\right)\right],
\end{aligned}
$$

and the result is now clear.

Lemma 7. If the sequence $\left(\beta_{n}\right)$ decreases to zero fast enough (more precisely if $\left(\sum_{n+1}^{\infty} \beta_{i}\right) / \beta_{n} \rightarrow 0$ as $\left.n \rightarrow \infty\right)$, the series

$$
\sum_{i=1}^{\infty} \beta_{i}\left(g_{i}-G_{0}\right)
$$


defines an element $g$ of $E^{*}$ which does not attain a maximum on $C$.

Proof. Assume to start with only that $\sum \beta_{i}$ converges. Now $K_{1}$, hence also $K_{1}-G_{0}$, is equicontinuous; hence there is a neighbourhood $U$ of 0 in $E$ such that

$$
x \in U \Rightarrow|f(x)| \leqq 1 \quad \text { for all } f \in K_{1}-G_{0} .
$$

Hence

$$
x \in U \Rightarrow \sum_{i=1}^{\infty} \beta_{i}\left[g_{i}(x)-G_{0}(x)\right] \leqq \sum_{i=1}^{\infty} \beta_{i} .
$$

This shows that $g$ is defined and continuous on $E$, i.e. $g \in E^{*}$. Now by the note (iii) after the definition of $p$, there is $M \geqq 0$ such that

$$
x \in C, \quad f \in K_{1}-G_{0} \Rightarrow|f(x)| \leqq M .
$$

Suppose that $g$ attains its supremum on $C$ at some point $u$ of $C$. Then for each $n$, we have

$$
\begin{aligned}
\sum_{1}^{n} \beta_{i}\left(g_{i}-G_{0}\right)(u)= & g(u)-\sum_{n+1}^{\infty} \beta_{i}\left(g_{i}-G_{0}\right)(u) \geqq g(u)-M \sum_{n+1}^{\infty} \beta_{i} \\
= & p(g)-M \sum_{n+1}^{\infty} \beta_{i} \geqq p\left[\sum_{1}^{n} \beta_{i}\left(g_{i}-G_{0}\right)\right] \\
& -p\left[\sum_{1}^{n} \beta_{i}\left(g_{i}-G_{0}\right)-g\right]-M \sum_{n+1}^{\infty} \beta_{i} \\
\geqq & p\left[\sum_{1}^{n} \beta_{i}\left(g_{i}-G_{0}\right)\right]-2 M \sum_{n+1}^{\infty} \beta_{i} \\
> & \frac{1}{2} \beta_{n} r+p\left[\sum_{1}^{n-1} \beta_{i}\left(g_{i}-G_{0}\right)\right]-2 M \sum_{n+1}^{\infty} \beta_{i} \\
\geqq & \frac{1}{2} \beta_{n} r+\sum_{1}^{n-1} \beta_{i}\left(g_{i}-G_{0}\right)(u)-2 M \sum_{n+1}^{\infty} \beta_{i} .
\end{aligned}
$$

Hence

$$
\left(g_{n}-G_{0}\right)(u)>\frac{1}{2} r-2 M\left(\sum_{n+1}^{\infty} \beta_{i}\right) / \beta_{n} .
$$

If we choose $\left(\beta_{n}\right)$ to decrease fast enough, for instance $\beta_{n}=1 / n$ !, we find that $\lim \inf \left(g_{n}-G_{0}\right)(u) \geqq \frac{1}{2} r$, which contradicts the fact that 
$\lim \inf g_{n}(u) \leqq G_{0}(u)$. Hence $g$ cannot attain its supremum on $C$ at any point of $C$, and the theorem is proved.

The author's thanks are due to Professor James for providing a copy of his proof and some related papers on weak compactness that have recently been, or are due to be published.

\section{BIBLIOGRAPHY}

1. R. C. James, Weakly compact sets, Trans. Amer. Math. Soc. 13 (1964), 129-140.

2. J. L. Kelley and I. Namioka, Linear topological spaces, Van Nostrand, Princeton, N. J., 1963.

3. W. F. Eberlein, Weak compactness in Banach spaces, Proc. Nat. Acad. Sci. U.S.A. 33 (1947), 51-53.

4. R. C. James, Characterizations of reflexivity, Studia Math. 23 (1964), 205-216.

University of NewCASTLE UPON TyNe, ENGLaNd 